\title{
Hepatocyte-specific mutation of both NF-kB RelA and STAT3 abrogates the acute phase response in mice
}

\author{
Lee J. Quinton, ${ }^{1}$ Matthew T. Blahna, ${ }^{1}$ Matthew R. Jones, ${ }^{1}$ Eri Allen, ${ }^{1}$ Joseph D. Ferrari, \\ Kristie L. Hilliard,1 Xiaoling Zhang, ${ }^{1}$ Vishakha Sabharwal, ${ }^{2}$ Hana Algül, ${ }^{3}$ Shizuo Akira, ${ }^{4}$ \\ Roland M. Schmid, ${ }^{3}$ Stephen I. Pelton,, ${ }^{1,2}$ Avrum Spira, ${ }^{1}$ and Joseph P. Mizgerd ${ }^{1}$

\begin{abstract}
1The Pulmonary Center, Boston University School of Medicine, Boston, Massachusetts, USA. ²Pediatric Infectious Disease Boston Medical Center, Boston, Massachusetts, USA. ${ }^{3}$ Technical University of Munich, Munich, Germany.

${ }^{4}$ Laboratory of Host Defense, Immunology Frontier Research Center, Osaka University, Osaka, Japan.
\end{abstract}

\begin{abstract}
The acute phase response is an evolutionarily conserved reaction in which physiological stress triggers the liver to remodel the blood proteome. Although thought to be involved in immune defense, the net biological effect of the acute phase response remains unknown. As the acute phase response is stimulated by diverse cytokines that activate either NF- $\mathrm{KB}$ or STAT3, we hypothesized that it could be eliminated by hepatocyte-specific interruption of both transcription factors. Here, we report that the elimination in mice of both NF-KB p65 (RelA) and STAT3, but neither alone, abrogated all acute phase responses measured. The failure to respond was consistent across multiple different infectious, inflammatory, and noxious stimuli, including pneumococcal pneumonia. When the effects of infection were analyzed in detail, pneumococcal pneumonia was found to alter the expression of over a thousand transcripts in the liver. This outcome was inhibited by the combined loss of RelA and STAT3. Moreover, this interruption of the acute phase response increased mortality and exacerbated bacterial dissemination during pneumonia, possibly as a result of acute humoral enhancement of macrophage opsonophagocytosis, which was impaired in the mutant mice. Thus, we conclude that RelA and STAT3 are essential for stress-induced transcriptional remodeling in the liver and the subsequent activation of the acute phase response, whose functional role includes compartmentalization of local infection.
\end{abstract}

\section{Introduction}

The acute phase response is defined by altered concentrations of select blood proteins under duress (1). It is evolutionarily conserved among animals with circulatory systems and applies broadly, with blood protein changes correlating with severity across many diseases. Individual acute phase proteins limit inflammation, which can prevent injury $(2,3)$ but exacerbate infection (4). Conversely, other acute phase proteins have antibacterial activities that decrease infection (5). However, the acute phase response is not defined by the activities of individual proteins, but rather by the net biological consequences of their changes. Effects of the acute phase response on the conditions with which it associates are speculative, as it has never been effectively or specifically interrupted.

Hepatocytes are the predominant source of acute phase proteins in the blood (1), with induction dependent on IL- 6 and the early response cytokines TNF- $\alpha$ and IL-1 (6-8), which activate STAT3 and NF-кB in the liver (7-10). The interruption of IL-6, the gp130 signaling receptor for IL-6, or STAT3 decreases but does not eliminate acute phase protein induction $(7,10,11)$. Roles of NF- $\kappa B$ in the acute phase response are inferred from associative and in vitro analyses $(7,9)$. We hypothesized that the hepatocyte-specific mutation of both STAT3 and NF-кB p65 (RelA) together would render the liver unresponsive to IL- 6 , TNF- $\alpha$, and IL- 1 , thereby ablating the acute phase response.

Conflict of interest: The authors have declared that no conflict of interest exists. Citation for this article: J Clin Invest. 2012;122(5):1758-1763. doi:10.1172/JCI59408.

\section{Results and Discussion}

To address the individual contributions of STAT3 or RelA, induction of acute phase protein transcripts during Streptococcus pneumoniae pneumonia was assessed in mutant mice lacking functional alleles for either transcription factor. The effects of each individual deletion were variable and incomplete (Supplemental Figure 1; supplemental material available online with this article; doi:10.1172/JCI59408DS1), as observed in other settings $(10,12)$. Therefore, neither mutation could abrogate induction of even this limited panel of acute phase proteins.

To address combined roles of STAT3 and RelA in hepatocytes, we mutated both together (Figure 1A). Mutant progeny were born at Hardy-Weinberg equilibrium and demonstrated no gross or histological abnormalities. RelA and STAT3 were nearly undetectable in mutant livers (Figure 1B), with residual immunoreactivity likely originating from nonhepatocytes. Neither protein was altered in other organs, indicating effective and accurate targeting.

In contrast to single transcription factor mutations (Supplemental Figure 1), simultaneously targeting both STAT3 and RelA completely eliminated hepatic mRNA induction of serum amyloid A 1 (SAA1), SAA2, serum amyloid P (SAP), and LPS-binding protein (LBP) during pneumococcal pneumonia (Figure 1C and Supplemental Figure 2). We also measured acute phase responses elicited by Gram-negative bacterial pneumonia, intravenous cytokines, or subcutaneous casein. Under every condition, combined STAT3 and RelA mutation ablated the induction of these acute phase proteins (Figure 1C). 
A
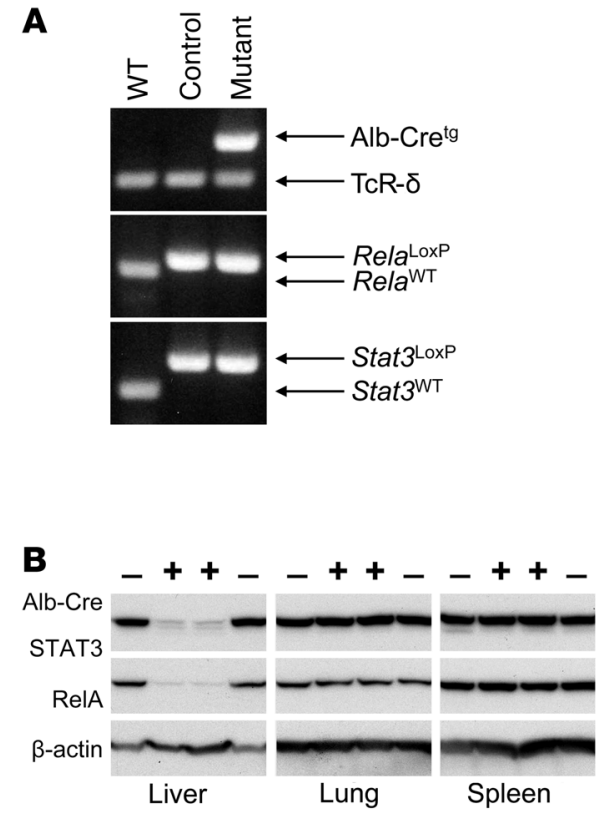

C
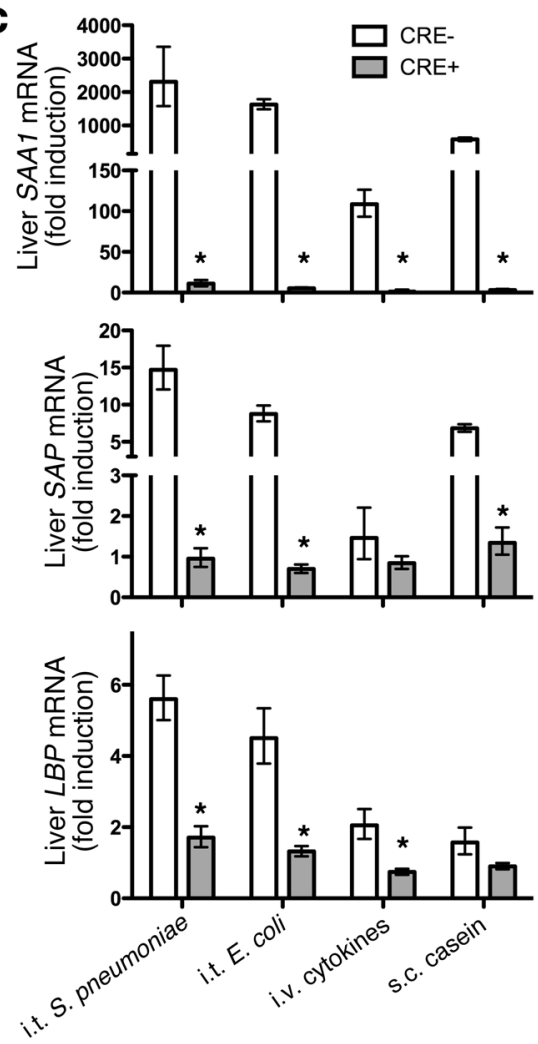

D
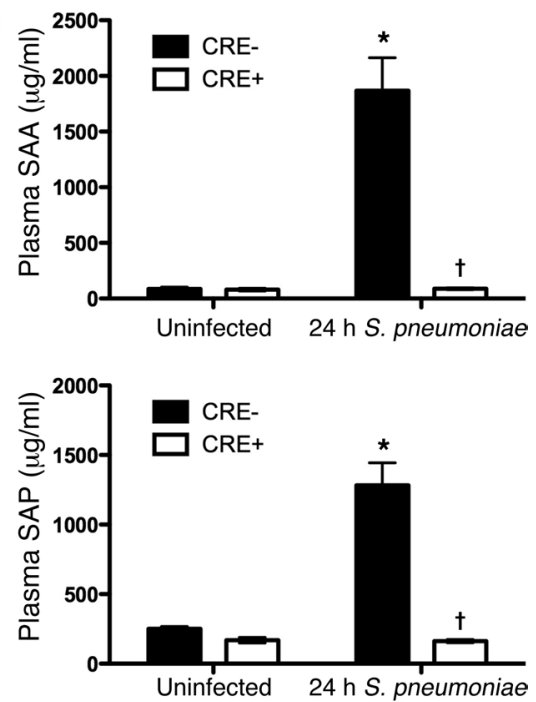

Figure 1

Deletion of STAT3 and RelA in hepatocytes eliminates acute phase protein induction. (A) PCR confirmed gene incorporations of Cre-recombinase driven by an albumin promoter (Alb-Cretg) and LoxP insertions in Rela and Stat3 alleles. TCR- $\delta$ was an amplification control. (B) Immunoblots revealed liver-specific deletion of STAT3 and RelA in CRE+ mice. (C) qRT-PCR was performed to determine Saa1, Sap, and Lbp mRNA induction in response to intratracheal (i.t.) S. pneumoniae (serotype 3; $10^{6} \mathrm{CFU}$ ), i.t. E. coli (106 CFU), i.v. cytokines (TNF- $\alpha$, IL-1 $\beta$, and IL-6), or s.c. casein. Values represent fold induction compared with that in uninfected CRE- mice, expressed as geometric means \pm geometric SEM. ${ }^{*} P<$ 0.05 ( $n=3-10$ mice/group). (D) Plasma concentrations of SAA and SAP were quantified in samples collected 24 hours after i.t. S. pneumoniae using ELISA and expressed as mean \pm SEM $\left(n=3-11\right.$ mice/group). ${ }^{*} P<0.05$, effect of infection; ${ }^{\dagger} P<0.05$, effect of genotype.

In the absence of infection, SAA and SAP were abundant in the blood and unaltered by hepatocyte targeting (Figure 1D). During pneumonia, plasma concentrations of SAA and SAP increased in controls, reaching $\mathrm{mg} / \mathrm{ml}$ quantities (Figure $1 \mathrm{D}$ ), potentially from expression in infected tissues and/or hepatocytes $(7,12)$. In hepatocyte mutants, blood concentrations of SAA and SAP were completely unchanged by pneumonia (Figure 1D). Thus, while baseline levels of these proteins are maintained by other pathways, blood acute phase changes are entirely dependent upon STAT3 and RelA in hepatocytes.

To comprehensively assess the influence of STAT3 and RelA deficiency, we profiled liver transcriptomes. In uninfected mice, only a single gene, Saa2, was significantly influenced by mutations, and this difference did not affect circulating SAA (Figure 1D), nor was it significant by quantitative RT-PCR (qRT-PCR) with larger sample sizes (Supplemental Figure 2). Therefore, baseline synthesis of liver mRNAs does not depend on these transcription factors, and there was no evidence for off-target effects from the Cre transgene. Confirming this, microarrays from pneumonic nonfloxed mice revealed that not a single transcript was significantly affected by hepatic Cre expression in the absence of RelA or STAT3 mutation (GEO GSE35516). During pneumonia, 1,157 targets were altered in control mice, indicating substantial remodeling of the hepatic transcriptome. Gene Ontology (GO) analyses revealed 26 biological processes altered during pneumonia (Table 1), including "acute-phase response" and categories related to protein synthesis, transport, and localization. Most genes affected by pneumonia did not encode acute phase proteins themselves, but supported their synthesis and secretion (Table 1). Remarkably, a mere 77 transcripts were affected by pneumonia in mutants, with GO analyses indicating no biological processes as significant (Table 1). A heat map including all transcripts affected by pneumonia in the control mice revealed a distinctly compromised response in the mutants (Figure 2A). Overall, 96\% of the mRNAs altered by pneumonia in control mice (positively or negatively) showed no significant changes in mutants, demonstrating a transcriptome-wide dependence of acute phase responses on STAT3 and RelA (Figure 2B).

Of known acute phase proteins (1), 25 transcripts were significantly changed by pneumonia in controls, of which only 3 were significant in double mutants (Supplemental Table 1). For these 

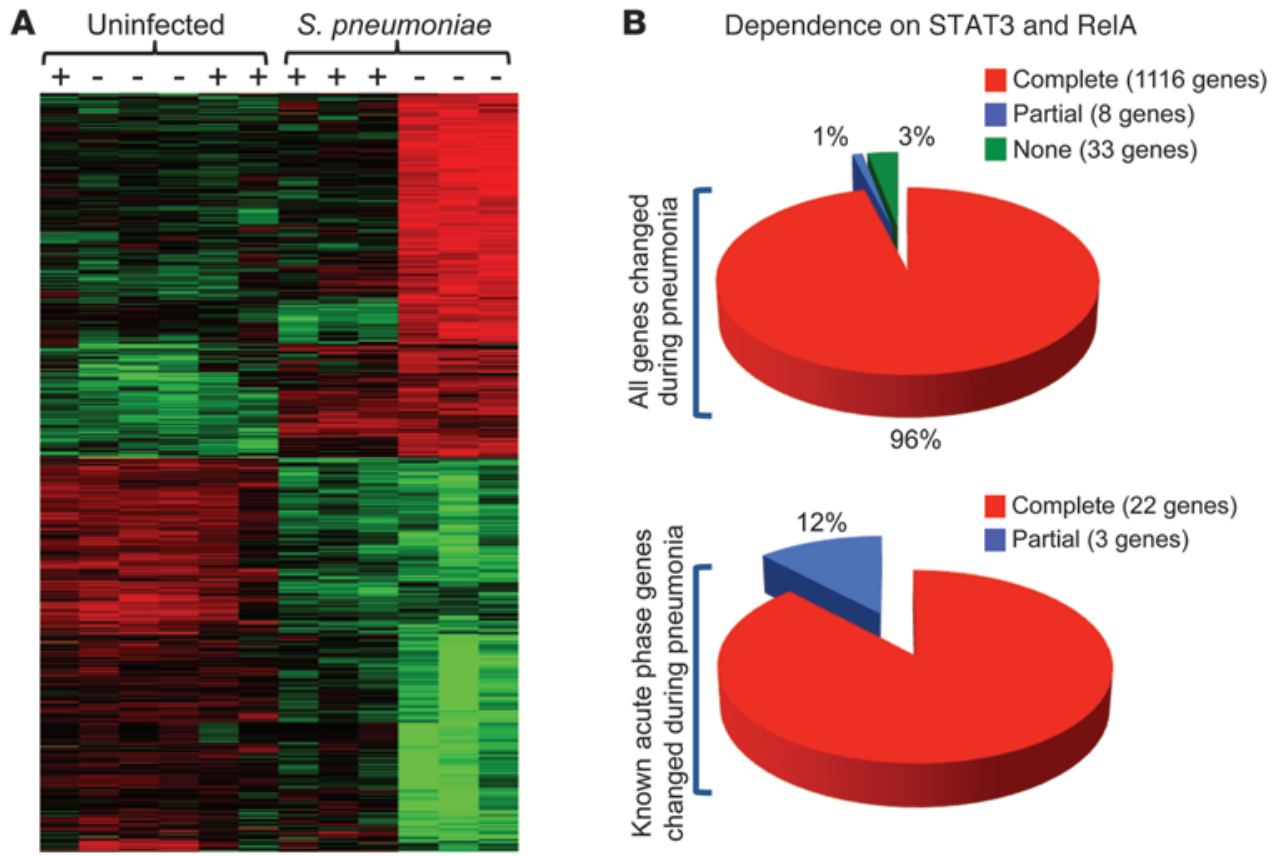

\section{Figure 2}

STAT3 and RelA in hepatocytes are required for the liver transcriptional response to pneumonia. (A) Heat map indicates relative gene expression for all 1,157 transcripts significantly $(F D R<0.05)$ changed in control mouse livers following 24 hours of pneumococcal pneumonia (serotype $\left.3 ; 10^{6} \mathrm{CFU}\right)$. Each column represents an individual control (-) or mutant (+) mouse, and each row is a distinct transcript. Red or green shows increased or decreased expression, respectively. (B) The effect of STAT3 and RelA deletion on pneumonia-influenced genes is indicated, using FDR $<0.05$ to differentiate transcripts that are: (a) completely dependent (significantly changed during pneumonia only in CRE- mice); (b) partially dependent (changed during pneumonia in both genotypes, but significantly less so in $\mathrm{CRE}^{+}$mice); and (c) independent (changed during pneumonia in both genotypes, with no significant difference between genotypes).

3 (Saa1, Saa2, and Lcn2), follow-up analyses using qRT-PCR and larger sample sizes revealed induction only in control mice (Supplemental Figure 2). Furthermore, plasma concentrations of SAA (including both SAA1 and SAA2) and lipocalin 2 (encoded by Lcn2) exhibited acute phase changes only in control mice (Figure $1 \mathrm{D}$ and Supplemental Figure 4). Thus, $100 \%$ of the known acute phase proteins altered during pneumonia require hepatocyte STAT3 or RelA.

Because any secreted protein from the liver whose expression changes during pneumonia may be an acute phase protein, we identified potentially secreted proteins in our data set. In addition to proteins listed in Supplemental Table 1, another 149 potentially secreted proteins were identified as changed during pneumonia (Supplemental Table 2). Of these, 97\% remained unchanged in the mutant mice (Supplemental Table 2), expanding the scope of the hepatic acute phase response.

Approximately half of the gene changes induced by pneumonia were decreases (599/1157 and 35/77 transcripts in controls and mutants, respectively). The biological processes involved (Table 1) included both positive and negative changes, all of which were absent in mutants. Of known negative acute phase proteins, the only one significantly changed during pneumonia (transthyretin) was not altered in mutants (Table 1). Thus, STAT3 and RelA are requisite for negative and positive acute phase changes. Similar microarray analyses were performed with livers from the individual STAT3 or RelA mutants with pneumonia. While 882 expressed targets were significantly altered in pneumonic double mutants compared with controls, only 110 were altered by deletion of STAT3 alone (Supplemental Figure 3), and none were significantly altered in microarray analyses of mice lacking RelA alone (Supplemental Figure 3). These data strongly support acute phase changes depending on either STAT3 or RelA. Mutation of both is necessary to ablate the hepatic acute phase response.

We used this double-mutant model to determine the significance of acute phase responses during pneumococcal pneumonia, where it was first discovered (13). Pneumonia is a public health priority, with major gaps in immunological understanding $(14,15)$. Mice were infected with a virulent serotype 3 isolate of $S$. pneumoniae, which grew in the lungs by 4 orders of magnitude in both genotypes (Supplemental Figure 5A). Histopathology, emigrated neutrophils, and cytokine synthesis were mostly unaffected by genotype (Supplemental Figure 6). In contrast, the invasiveness of pneumococcal infection was significantly exaggerated by the absence of an acute phase response. The hepatocyte mutations doubled the likelihood of bacteremia, with 81\% of mutants becoming bacteremic versus $39 \%$ of controls (Figure 3A). Splenic bacteria were also elevated in mutants (Supplemental Figure 5B). Bacteremia correlated with lung pneumococcal burden in control mice but not mutants (Supplemental Figure 7), suggesting that compromised systemic immunity was distinct from pulmonary host defenses. Effects were also seen with a less virulent serotype $19 \mathrm{~F}$ isolate (16), as mice without acute phase responses showed increased mortality (Figure 3B). Thus, 
Table 1

Categories of biological processes significantly represented by transcripts altered in the livers of $\mathrm{CRE}^{-}$control mice after 24 hours of pneumococcal pneumonia

GO ID
GO:0015031
GO:0045184
GO:0008104
GO:0033036
GO:0008152
GO:0048193
GO:0006888
GO:0006886
GO:0006066
GO:0006953
GO:0019752
GO:0006082
GO:0008202
GO:0006810
GO:0051234
GO:0006487
GO:0046907
GO:0051641
GO:0019318
GO:0046903
GO:0009058
GO:0009611
GO:0005996
GO:0051649
GO:0044262
GO:0006950

\author{
GO Category (CRE-) \\ Protein transport \\ Establishment of protein localization \\ Protein localization \\ Macromolecule localization \\ Metabolic process \\ Golgi vesicle transport \\ ER to Golgi vesicle-mediated transport \\ Intracellular protein transport \\ Alcohol metabolic process \\ Acute-phase response \\ Carboxylic acid metabolic process \\ Organic acid metabolic process \\ Steroid metabolic process \\ Transport \\ Establishment of localization \\ Protein amino acid N-linked glycosylation \\ Intracellular transport \\ Cellular localization \\ Hexose metabolic process \\ Secretion \\ Biosynthetic process \\ Response to wounding \\ Monosaccharide metabolic process \\ Establishment of cellular localization \\ Cellular carbohydrate metabolic process \\ Response to stress
}

No categories were significantly represented in $\mathrm{CRE}^{+}$mice.

beyond being a biomarker, altered blood concentrations of liverderived acute phase proteins make essential contributions to host defense, protecting the blood and other organs from disseminating microbes.

To determine whether altered protein concentrations enhanced humoral bacteriostatic or bactericidal activities, we quantified the survival and growth of pneumococcus in sera from pneumonic mice. Across a variety of serum concentrations, growth curves were similar between genotypes (Supplemental Figure 8). Blood protein changes mediated by hepatocyte transcription factors do not directly influence the multiplication or survival of pneumococcus.

We hypothesized that changes in circulating liver-derived proteins augmented macrophage opsonophagocytosis (5, 17). Serum from infected control mice significantly enhanced phagocytosis compared with that from uninfected mice (Figure $3 \mathrm{C}$ ). In the absence of infection, there was no effect of genotype (data not shown). However, during pneumonia, serum from mutants was significantly less effective than that from WT mice (Figure 3D). Serum from pneumonic mutants was also less capable of labeling bacteria with complement C3 (Figure $3 \mathrm{E})$. Although C3 was not induced during pneumonia (Supplemental Table 1 and data not shown), deposition of C3 is likely influenced by other changes in pneumonic serum. For instance, SAP, significantly reduced in mutant plasma (Figure 1D), mediates C3 deposition on pneumococcus (5). Thus, the acute phase changes driven by hepatocyte STAT3 and RelA are essential for

\section{Significance (FDR)}

$1.20 \mathrm{E}-07$

$1.76 \mathrm{E}-06$

$5.02 \mathrm{E}-06$

$1.28 \mathrm{E}-05$

$3.76 \mathrm{E}-05$

$1.02 \mathrm{E}-04$

$1.50 \mathrm{E}-04$

1.67E-04

$2.41 \mathrm{E}-04$

$5.53 \mathrm{E}-04$

$5.94 \mathrm{E}-04$

$6.27 \mathrm{E}-04$

9.53E-04

0.001080684

0.001724968

0.001951214

0.002719528

0.020586554

0.022188765

0.028444699

0.028877296

0.029395074

0.03028701

0.034332905

0.040265459

0.048067635 remodeling the blood proteome to enhance macrophage-mediated host defense.

We conclude by proposing hepatocyte mutation of both STAT3 and RelA as a specific and effective inhibition of the acute phase response. All acute phase proteins are affected. The response to duress is ablated, while basal expression is not. Only hepatocytes are targeted, leaving cells in other tissues (e.g., the site of injury or infection) intact. During pneumonia, acute phase changes in liver-derived blood proteins are critical for enhancing opsonophagocytosis of bloodborne pathogens. Since hepatic STAT3 and RelA activity depend on upstream signaling from IL-6 and early-response cytokines during pneumonia (7), we posit that these signaling intermediates constitute an integrated axis of systemic innate immunity, with lung-derived cytokines triggering changes in acute phase protein transcription in the liver to form a vascular shield against disseminated infection (Supplemental Figure 9). Systemic antibacterial innate immunity is likely but one role of the blood proteome remodeling that occurs during disease, with additional roles yet to be discovered.

\section{Methods}

Additional information is in Supplemental Methods.

Mice. Mice that were Stat $3^{\operatorname{Lox} P / L o x P}(18)$ or Rela ${ }^{\text {LoxP/LoxP }}$ (19) were crossed with $\mathrm{Alb}$-Cretg/tg mice expressing Cre-recombinase from an albumin promoter (20) to generate mice lacking STAT3 (Alb-Cre ${ }^{\text {tg }} / /$ Stat 3 LoxP/LoxP $)$, RelA

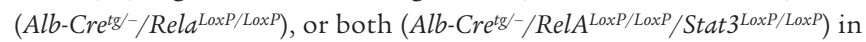
hepatocytes. Alb-Cretg tg mice were on a C57BL/6 background, matching that of control mice when applicable. Other mice contained a mixed genetic background, with results from $\mathrm{CRE}^{+}$mutants compared with littermates with identical LoxP insertions but no CRE transgene. Intratracheal instillations of bacteria were as described (7), using S. pneumoniae serotype 3 (ATCC 6303), S. pneumoniae serotype 19F (EF3030; provided by Marc Lipsitch, Harvard University, Cambridge, Massachusetts, USA), or E. coli serotype 06:K2:H1 (ATCC 19138). Other mice were administered $0.5 \mathrm{ml}$ of $10 \%$ casein s.c. (MP Biomedicals) or $100 \mu \mathrm{l}$ of a mixture containing 200 ng each IL-6, TNF- $\alpha$, and IL- $1 \beta$ i.v. (R\&D Systems).

Microarray. Microarrays were performed on total liver RNA as described in figure legends. All data were deposited in the NCBI Gene Expression Omnibus (GEO GSE35516).

Statistics. Statistical analyses were performed using GraphPad Prism 5.0. Comparisons between 2 groups were performed using a 2-tailed unpaired Student's $t$ test, paired Student's $t$ test, or Mann-Whitney $U$ test. Multiple groups were compared using a 2-way ANOVA followed by a Bonferroni's post-test. To control for multiple comparisons in microarray data, false 

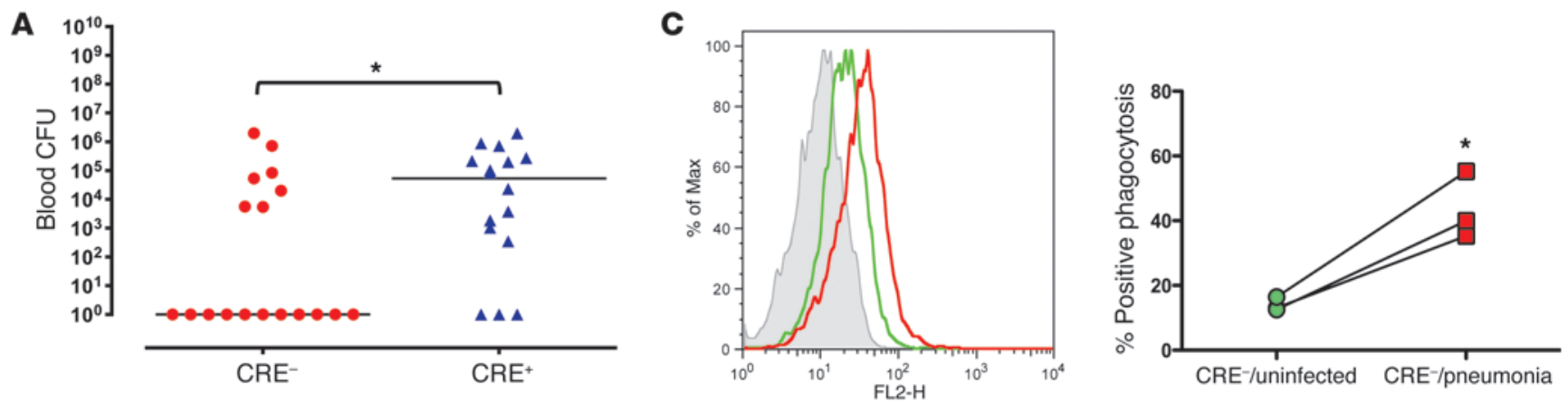

\section{B}
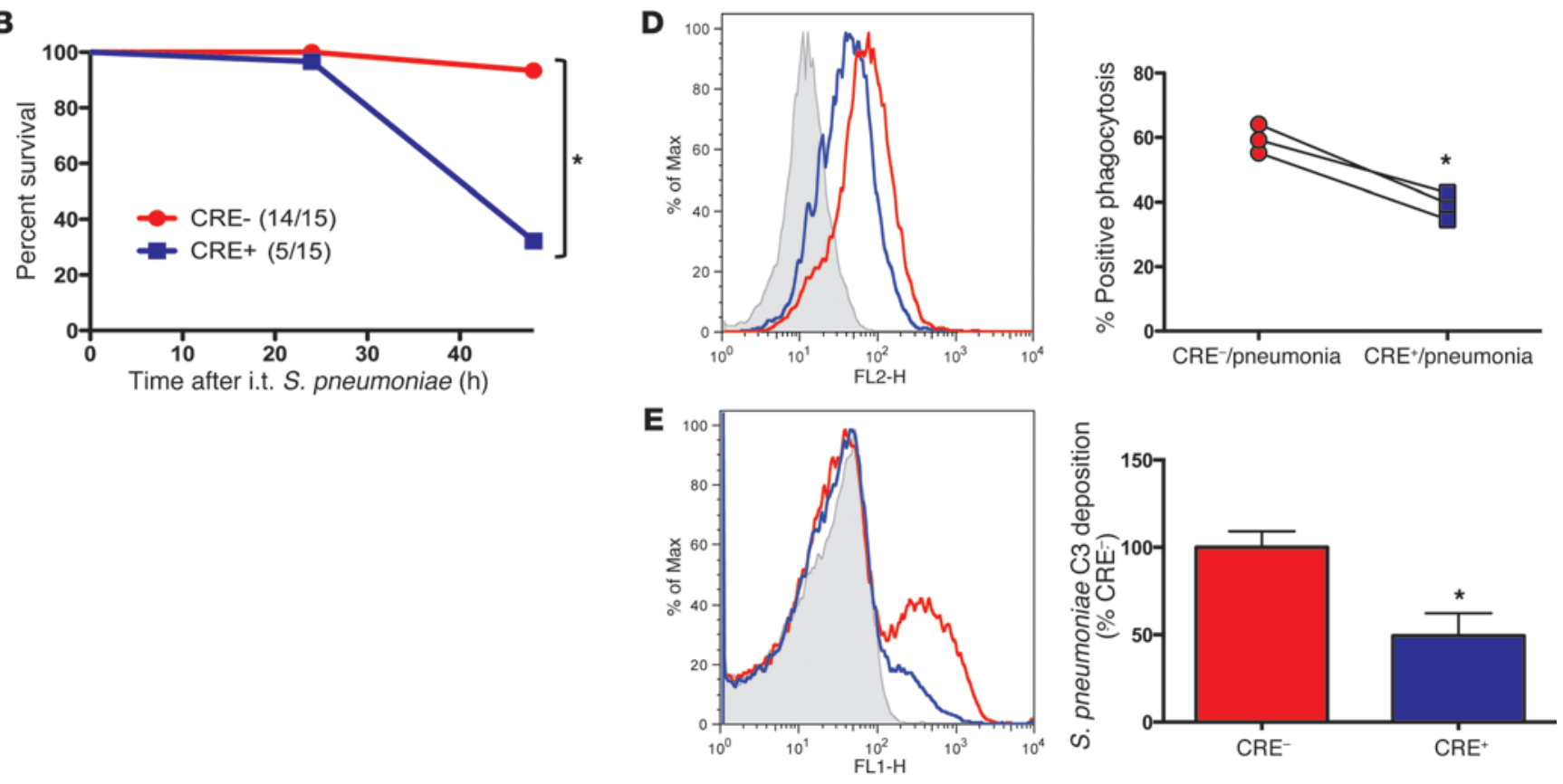

\section{Figure 3}

Liver STAT3 and RelA are required for blood-borne host defense during pneumonia. (A) Living bacteria were enumerated in blood collected 48 hours after intratracheal S. pneumoniae serotype $3\left(10^{4} \mathrm{CFU}\right)$. Data points represent individual mice, and lines indicate medians $(n=16-18)$. (B) Survival through 48 hours was documented after intratracheal instillation of $S$. pneumoniae serotype $19 F\left(10^{6}\right.$ CFU) $(n=15)$. (C and D) Effects of serum on opsonophagocytosis were measured by quantifying fluorescence/cell using flow cytometry after J774A.1 mouse macrophage-like cells were incubated with fluorescent $S$. pneumoniae and mouse serum. Representative histograms and the percentage of cells fluorescent illustrate effects of (C) pneumonia (in control CRE- mice) or (D) genotype (during pneumonia). Results represent 3 experiments, each containing pooled sera from distinct mice. (E) C3 deposition was measured on serum-opsonized S. pneumoniae by flow cytometry. Data represent mean \pm SEM of the percentage of $\mathrm{C}^{+}$bacteria exposed to serum from different mice $(n=4-7)$. Colors in $\mathbf{C}-\mathbf{E}$ correspond to serum collected from uninfected mice (green), 24-hour infected $\mathrm{CRE}^{-}$control mice (red), or 24-hour infected CRE+ mutant mice (blue). Shaded curves represent background (nonopsonized) bacterial fluorescence (E) or background J774A.1 fluorescence (C and D), which was similar for cells alone, cells exposed to nonopsonized bacteria, or experiments conducted at $0^{\circ} \mathrm{C}$. ${ }^{*} P<0.05$, difference between groups.

discovery rate (FDR) was calculated. Significance of correlation was determined using the Pearson's $r$ test. Survival data were analyzed using a log-rank (Mantel-Cox) test. Comparisons were considered significant with a $P$ value or FDR of less than 0.05 .

Study approval. Protocols were approved by the Institutional Animal Care and Use Committee at Boston University.

\section{Acknowledgments}

This work was supported by NIH R00-HL092956, NIH R01HL079392, NIH R01-HL068153, the Deutsche Forschungsgemeinschaft (DFG AL 1174/3-1; INST 95/613-3/TP A10), and a Parker
B. Francis Fellowship. We thank the Boston University School of Medicine microarray core and the Clinical and Translational Science Institute (NIH 1UL1RR025771) for technical assistance.

Received for publication June 8, 2011, and accepted in revised form February 15, 2012.

Address correspondence to: Joseph P. Mizgerd or Lee J. Quinton, 72 E. Concord St., Boston, Massachusetts 01760, USA. Phone: 617.638.4860; Fax: 617.536.8093; E-mail: jmizgerd@bu.edu (J.P. Mizgerd), lquinton@bu.edu (L.J. Quinton). 
1. Gabay C, Kushner I. Acute-phase proteins and other systemic responses to inflammation. NEnglJ Med. 1999;340(6):448-454.

2. Deban L, et al. Regulation of leukocyte recruitment by the long pentraxin PTX3. Nat Immunol. 2010;11(4):328-334.

3. Sander LE, et al. Hepatic acute-phase proteins control innate immune responses during infection by promoting myeloid-derived suppressor cell function. J Exp Med. 2010;207(7):1453-1464.

4. Renckens R, Roelofs JJ, Knapp S, de Vos AF, Florquin $S$, van der Poll $T$. The acute-phase response and serum amyloid $\mathrm{A}$ inhibit the inflammatory response to Acinetobacter baumannii Pneumonia. J Infect Dis. 2006;193(2):187-195.

5. Yuste J, Botto M, Bottoms SE, Brown JS. Serum amyloid P aids complement-mediated immunity to Streptococcus pneumoniae. PLoS Pathog. 2007;3(9):1208-1219.

6. Zheng $\mathrm{H}$, et al. Resistance to fever induction and impaired acute-phase response in interleukin-1 beta-deficient mice. Immunity. 1995;3(1):9-19.

7. Quinton LJ, Jones MR, Robson BE, Mizgerd JP. Mechanisms of the hepatic acute-phase response during bacterial pneumonia. Infect Immun.
2009;77(6):2417-2426

8. Kopf $M$, et al. Impaired immune and acute-phase responses in interleukin-6-deficient mice. Nature. 1994;368(6469):339-342.

9. Betts JC, Cheshire JK, Akira S, Kishimoto T, Woo P. The role of NF-kappa B and NF-IL6 transactivating factors in the synergistic activation of human serum amyloid A gene expression by interleukin-1 and interleukin-6. J Biol Chem. 1993;268(34):25624-25631.

10. Sakamori R, et al. Signal transducer and activator of transcription 3 signaling within hepatocytes attenuates systemic inflammatory response and lethality in septic mice. Hepatology. 2007;46(5):1564-1573.

11. Dierssen U, et al. Molecular dissection of gp130dependent pathways in hepatocytes during liver regeneration. J Biol Chem. 2008;283(15):9886-9895.

12. Alonzi T, Maritano D, Gorgoni B, Rizzuto G, Libert C, Poli V. Essential role of STAT3 in the control of the acute-phase response as revealed by inducible gene inactivation [correction of activation] in the liver. Mol Cell Biol. 2001;21(5):1621-1632.

13. Tillett WS, Francis T. Serological reactions in pneumonia with non-protein somatic fraction of pneumococcus. J Exp Med. 1930;52(4):561-571.

14. Mizgerd JP. Lung infection--a public health prior- ity. PLoS Med. 2006;3(2):e76.

15. Mizgerd JP. Acute lower respiratory tract infection. N Engl J Med. 2008;358(7):716-727.

16. Jones MR, Simms BT, Lupa MM, Kogan MS, Mizgerd JP. Lung NF-kappaB activation and neutrophil recruitment require IL-1 and TNF receptor signaling during pneumococcal pneumonia. J Immunol. 2005;175(11):7530-7535.

17. Lu J, Marnell LL, Marjon KD, Mold C, Du Clos TW, Sun PD. Structural recognition and functional activation of FcgammaR by innate pentraxins. Nature. 2008;456(7224):989-992.

18. Takeda K, Kaisho T, Yoshida N, Takeda J, Kishimoto T, Akira S. Stat3 activation is responsible for IL-6-dependent T cell proliferation through preventing apoptosis: generation and characterization of T cell-specific Stat3-deficient mice. J Immunol. 1998;161(9):4652-4660.

19. Algul H, et al. Pancreas-specific RelA/p65 truncation increases susceptibility of acini to inflammation-associated cell death following cerulein pancreatitis. J Clin Invest. 2007;117(6):1490-1501.

20. Postic C, Magnuson MA. DNA excision in liver by an albumin-Cre transgene occurs progressively with age. Genesis. 2000;26(2):149-150. 